\title{
antioxidants
}

ISSN 2076-3921

www.mdpi.com/journal/antioxidants

Review

\section{Antioxidant and Antiradical Activity of Coffee}

Alexander Yashin ${ }^{1}$, Yakov Yashin ${ }^{1}$, Jing Yuan Wang ${ }^{2}$ and Boris Nemzer ${ }^{2, *}$

1 InterLab, Inc., Selskohozyaistvennaya 12a, Moscow 129226, Russia;

E-Mails: yashinchrom@mail.ru (A.Y.); yashin@interlab.ru (Y.Y.)

2 VDF FutureCeuticals, Inc., 2692 N. State Rt. 1-17, Momence, IL 60954, USA;

E-Mail: rwang@futureceuticals.com

* Author to whom correspondence should be addressed; E-Mail: bnemzer@futureceuticals.com; Tel.: +1-815-507-1400; Fax: +1-815-550-0013.

Received: 9 August 2013; in revised form: 27 September 2013 / Accepted: 29 September 2013 / Published: 15 October 2013

\begin{abstract}
This review summarizes published information concerning the determination of antioxidant activity (AA) in coffee samples by various methods (ORAC, FRAP, TRAP, TEAC, etc.) in vitro and limited data of antiradical activity of coffee products in vitro and in vivo. Comparison is carried out of the AA of coffee Arabica and coffee Robusta roasted at different temperatures as well as by different roasting methods (microwave, convection, etc.). Data on the antiradical activity of coffee is provided. The antioxidant activity of coffee, tea, cocoa, and red wine is compared. At the end of this review, the total antioxidant content (TAC) of coffee samples from 21 coffee-producing countries as measured by an amperometric method is provided. The TAC of green and roasted coffee beans is also compared.
\end{abstract}

Keywords: antioxidants; antioxidant activity; ORAC; amperometry; coffee

\section{Introduction}

Studies carried out in recent decades have confirmed that excessive accumulation of oxygen and nitrogen reaction products in body fluids including free radicals, such as the superoxide anion, hydroxyl radical, hydroperoxyl radical, etc., is a major cause of pathological changes in the human body, resulting in premature aging and numerous diseases.

The steady increase of free radicals in cells creates the conditions for so-called oxidative stress, wherein free radicals oxidize blood vessel walls, protein molecules, DNA, carbohydrates, and lipids. 
These radicals are particularly active in interacting with membrane lipids that contain unsaturated bonds, and thus alter the properties of cell membranes. The most active free radicals break bonds in DNA molecules and damage the cells' genetic apparatus regulating their growth, which can result in cancerous cells. Oxidized low-density lipoproteins can be deposited on blood vessel walls which leads to atherosclerosis and cardiovascular disease [1-3].

Oxidative stress also plays a key role in the pathogenesis of aging [4,5]. Oxidative stress can be caused by various negative impacts, such as gamma or UV radiation, environmental factors, polluted and poor-quality food, stress, some medications or treatments, smoking, alcoholism, etc.

Prolonged oxidative stress inevitably leads to dangerous diseases - such as cancer, cardiovascular diseases, or diabetes - and premature aging. Oxidative stress can be reduced by antioxidant therapy, i.e., by consumption of certain amounts of natural antioxidants contained in vegetables, fruits, berries, vegetable oils, honey, tea, coffee, cocoa, juices, wine, sprouted grains, and other foods [6-8]. However, in order to control consumption of antioxidants, it is necessary to know their content in foods and beverages. In this regard, quantitative measurement of antioxidants in foods and beverages and compilation of a corresponding databank becomes a highly important objective. Coffee is one of the major sources of antioxidants in people's daily diet.

\section{Review of Publications on Determination of Coffee Antioxidant Activity in Vitro}

Beneficial health effects of coffee are usually attributed to its high antioxidant activity (ability to inhibit the process of oxidation). Many publications provide comparison of the antioxidant activity in such popular beverages as coffee, tea, and cocoa [9-11].

Antioxidant activity of coffee is related to chlorogenic, ferulic, caffeic, and $n$-coumaric acids contained in it [12]. In roasted coffee, melanoidins (brown pigments) are synthesized - these are strong antioxidants [13]. In some publications, caffeine and trigonelline are considered to be antioxidants also [14]. Phenylalanines which are formed during the roasting process show high antioxidant activity [14], as do heterocyclic compounds [15].

Many publications explore the relationship between coffee antioxidant activity and roasting, for example:

-Comparison of antioxidant activity in green coffee beans with roasted coffee $[16,17]$;

-Dependence of antioxidant activity on roasting temperature and time [17];

-Relation between the antioxidant activity and roasting method used (convection and microwave roasting) [17].

Many researchers believe during the roasting process that Maillard reaction products, which are also strong antioxidants, are formed [12,16,17].

The antioxidant activity of volatile compounds, such as furans, pyrroles, formed by pyrolysis of coffee, has also been studied [14].

Interestingly, the compound, such as chlorogenic acid and polyphenols, which contributed to the antioxidant activity in coffee, is geographically related [18]. The coffee fruit was found to have more chlorogenic acids (CGA) in Arabica coffee fruit planted in Mexico and India compared to the coffee fruit grew in China. 
In addition, evidence indicates that extraction procedures could affect the antioxidants contents in coffee fruit as well as the caffeine content [19]. It has been shown that the antioxidant activity was high in coffee fruit extract with low caffeine concentration in comparison with coffee fruit powder.

Apart from coffee, the antioxidant activity after roasting was also measured in wheat, nuts, and some other food products.

The antioxidant activity of green and roasted coffee is compared in several publications [20-22].

\section{Methods of Measuring the Antioxidant Activity and Antioxidant Content in Coffee}

Many methods based on new reagents, model systems and devices were suggested to use for determining the antioxidant activity, and many reviews were published on this subject [23-27].

The antioxidant activity (AA) is measured by various chemical and physicochemical methods. All those methods are most often based on the direct or indirect measurement of reaction rate and/or completeness.

Three types of methods could be distinguished based on the following measurements:

-Oxygen intake;

-Formation of oxidation products;

-Uptake or binding of free radicals.

In the first and second cases, AA is determined based on the degree of inhibition or intake rate for reagents or the products formed.

Primary methods for the AA measurements are: ORAC-oxygen radical absorbance capacity; TRAP — total radical trapping antioxidant parameter; FRAP — ferric reducing antioxidant power; TEAC (Randox)-trolox equivalent antioxidant capacity; ABTS-2,2-azinobis(3-ethylbenzthiazoline)-6sulfonic acid; TBARS - thiobarbituric acid reactive substance.

In all of these methods, the AA depends on multiple parameters, including time, temperature, nature of the substance, concentration of the antioxidants and other compounds, etc.

The antioxidant activity cannot be measured directly — what is typically measured is the effect of the antioxidants on the degree of oxidation. All of these methods often give conflicting results.

The drawback of many methods used for measuring antioxidant activity is the lack of proper substrates during the measurement process. Antioxidant activity is most often measured based on long-living synthetic free radicals (ABTS, DPPH, AAPH, etc.). Lots of synonymous terms have been proposed, including "antioxidant ability", "antioxidant power", "antioxidant activity", and "antioxidant capacity" [28]. All of these terms are related to the antioxidant concentration (the activity of substances or substance groups).

Many well-known methods, such as TEAC, TRAP, FRAP, etc., are based on reduction reactions of long-living free radicals or Fe (III) complex.

Obviously, all these methods have drawbacks because they use synthetic free radicals which have nothing in common with free radicals in the human body.

The above methods are time-consuming and include several stages; the signals are recorded using expensive devices, such as spectrophotometric, fluorimetric, and chemiluminescent detectors. 
Electrochemical methods are more promising for the measurement of integral antioxidant capacity because the reaction between active oxygen compounds in aqueous media is accompanied by electron transfer, i.e., they are electrochemical in nature.

In [29] has been proposed to measure antioxidant capacity by using electrogenerated oxidizers: chlorine, bromine, and iodine. The electrogeneration of halogens during coulometric titration was performed at a constant current of $5.0 \mathrm{~mA}$ from $0.2 \mathrm{M}$ aqueous solutions of $\mathrm{KCl}$ and $\mathrm{KBr}$ in $0.1 \mathrm{M} \mathrm{H}_{2} \mathrm{SO}_{4}$; titration end-point was detected by amperometric indication using two polarized platinum electrodes. The authors of this method were the first to introduce a characteristic called "bromine-scavenging antioxidant capacity" which is expressed in electricity units (coulombs) used for the titration of $100 \mathrm{~g}$ (or $100 \mathrm{~mL}$ ) of the tested material by electrogenerated bromine.

It was demonstrated that the value of bromine-scavenging antioxidant capacity reflects the total content of antioxidants in foods, beverages, and extracts of medicinal plants. It should be noted, however, that bromine and iodine can oxidize not only the antioxidants but also other compounds.

In one publication, it has been proposed to measure antioxidant activity by a potentiometric method using a mediator solution of $\mathrm{K}_{3}\left[\mathrm{Fe}(\mathrm{CN})_{6}\right] / \mathrm{K}_{4}\left[\mathrm{Fe}(\mathrm{CN})_{6}\right]$ [28].

This method was successfully used for the determination of the antioxidant activity of pure chemical compounds as well foods, beverages, dietary supplements, and extracts of medicinal plants.

Another electrochemical method proposed is cathodic stripping voltammetry. In this method, oxygen electroreduction is used as a model reaction.

In order to determine antioxidants in the tested substances, the dependences of the first oxygen reduction peak current on their concentration in the solution and the duration of the process.

In this method, a mercury film electrode (banned in many countries) was used as an indicator.

Antioxidant activity of many foods and biological samples have been measured by this method.

\subsection{ORAC (Oxygen Radical Absorbance Capacity) Method}

The ORAC, one of the methods most often used for the determination of antioxidant activity, especially in the U.S., was proposed by Cao G. [30] and later has been automated and validated by Prior, R., Ou, B. and other four different assays [31-35].

This method is based on measuring changes in fluorescence intensity vs. reaction time. Quantitative measurement of the antioxidant activity is carried out by determining the area between two curves which represent reaction with and without the antioxidant.

In the early tests, B-phycoerythrin was used as the fluorescent probe, which tended to show slightly lower results because this protein reacted with polyphenols. That is why it was later suggested to rather use fluorescein, a more stable fluorescent compound.

In this method, 2,2'-azobis-(2-amidinopropane) dihydrochloride (AAPH) is used as the source of peroxyl radicals. In the ORAC method, trolox, a water-soluble analog of vitamin E, is used as the standard. Fluorescence intensity is measured at $515 \mathrm{~nm}$ with the excitation wavelength of $485 \mathrm{~nm}$.

Changes in the fluorescence intensity are registered every minute over $35 \mathrm{~min}$, both with and without an antioxidant. Therefore, the total measurement lasts for $70 \mathrm{~min}$. The results obtained for each sample are then calculated using calibration curves and presented in $\mathrm{mmol} / \mathrm{g}$ of dry matter. 
The overall mechanism of the ORAC method is as follows: In the solution, the AAPH reagent decomposes at $37{ }^{\circ} \mathrm{C}$ with the release of peroxide free radical. When an antioxidant, or a mixture of antioxidants, are added, fluorescence quenching decreases due to the fact that antioxidants neutralize the effects of free radicals on the fluorescein. The convergence of the method is in the form of standard deviation (SD) $\pm 15 \%$.

In a 2005, Prior, et al. [36] wrote that a standardized total antioxidant capacity method should evaluate "effectiveness against various ROS/RNS such as superoxide anion, hydroxyl, and peroxynitrite...and this may require additional methods specific for each radical source".

The new generation of ORAC assay-total ORAC for food and nutrition (Total ORAC FN) has been introduced by Brunswick Laboratories (Norton, MN, USA) in 2008. Total ORAC FN represents a breakthrough in comprehensive antioxidant testing for food and nutrition products as antioxidant activity against five of the most important free radicals found in human-hydroxyl (HORAC assay), peroxyl (ORAC assay), peroxynitrite (NORAC assay), singlet oxygen (SOAC assay), and superoxide anion (SORAC assay).

The total antioxidant capacity of whole coffee fruits Arabica and their extracts commercially produced by FutureCeuticals, Inc. (Momence, IL, USA) has been analyzed using Total ORAC FN assays [19]. The results of ORAC FN are shown in Table 1.

Table 1. Total ORAC activity coffee fruit sample [19]. (Note: ORAC FN results are expressed as $\mu$ mole Trolox Equivalent (TE) $/ g \pm \mathrm{SD}$.

\begin{tabular}{ccccc}
\hline Coffee Fruit Sample & Coffee Fruits Extract-1 & $\begin{array}{c}\text { Coffee Fruits } \\
\text { Extract-2 }\end{array}$ & $\begin{array}{c}\text { Coffee Fruits } \\
\text { Powder-1 }\end{array}$ & $\begin{array}{c}\text { Coffee Fruits } \\
\text { Powder-2 }\end{array}$ \\
\hline Extration/Drying & Multistep ethanol & Single step extraction & Freeze-dried & Air-dried \\
Procedure & extraction & $45.0 \%$ & $8.79 \%$ & $4.53 \%$ \\
Total CGA & $76.5 \%$ & $6097 \pm 225$ & $823 \pm 86$ & $735 \pm 47$ \\
ORAC & $15,246 \pm 453$ & $18,709 \pm 426$ & $3520 \pm 287$ & $2140 \pm 125$ \\
HORAC & $41,389 \pm 3447$ & $527 \pm 52$ & $75 \pm 11$ & $52 \pm 7$ \\
NORAC & $1317 \pm 104$ & $860 \pm 24$ & $271 \pm 14$ & $123 \pm 62$ \\
SORAC & $2193 \pm 1591$ & $2042 \pm 185$ & $311 \pm 13$ & $239 \pm 12$ \\
SOAC & $3422 \pm 355$ & $28,237 \pm 782$ & $4768 \pm 285$ & $3439 \pm 134$ \\
\hline
\end{tabular}

Table 1 shows that the total chlorogenic acid (CGA) contents correlates to total antioxidant capacity. The coffee extract with the highest chlorogenic acid contents corresponds to the highest total ORAC activity compared with whole coffee fruit powder (air dried and freeze dried) [19].

\subsection{Amperometric Method for Determining Total Antioxidant Content}

Amperometric method (AM) is based on measuring an electric current in the detector cell which occurs during oxidation of the analyte on the working electrode surface when certain potentials are applied. Thus, when an amperometric method is used, the changes in the current passing through the cell are registered - these changes directly correlate to the changes in the analyte concentration [1].

The amperometric method can operate in three modes: at a constant potential, at pulse potentials, and by scanning the potentials in the entire range. 
The amount of electric current depends on the nature of the analyte, nature of the working electrode, and the potential applied to the electrode.

Emerging electric currents are very small, within $10^{-6}$ to $10^{-10} \mathrm{~A}$. These analog signals are amplified, converted into digital signal by an analog-digital converter (ADC), and then displayed on a computer screen. If necessary, the outputs can be printed.

The working electrode is a glassy carbon electrode which is most commonly used in the determination of polyphenolic compounds. Ionization potentials of phenolic compounds vary within 100-1300 mV.

The signal is recorded as differential output curves. Using special software, the areas or peak heights (of the differential curves) are calculated for the analyte and for the reference substance. Well-known antioxidants, such as quercetin, dihydroquercetin, mexidol, trolox, gallic acid, etc. can be used as reference substances. The amperometric method has several advantages in the determination of antioxidant activity: not taking into account sample preparation, one determination takes only a few min; analysis (data recording and processing) takes place in real time; accuracy and reproducibility of the analysis is ensured by accurate dosing with a six-way valve; standard deviation (SD) of valve dispenses is less than $0.5 \%$; SD of the successive measurements of the analyzed samples is less than $5 \%$; limit of detection for polyphenols and flavonoids is at the level of nanograms and picograms $\left(10^{-9}-10^{-12} \mathrm{~g}\right)$. At such low concentrations, the likelihood of the mutual influence of different jointly present antioxidants, such as by a manifestation of synergy, is significantly reduced.

At the same time, high selectivity in determination only of antioxidants (compounds capable of inhibiting oxidation) is achieved; other compounds present in complex mixtures do not interfere with the determination of the antioxidants. No chemicals (other than standards) are required for this analysis; therefore, the cost of measurements is very low.

The amperometric method is the only method which allows for direct measurement of all antioxidants in a sample. The amperometric method has been successfully applied to determine the antioxidant capacity of various wines and coffee [37]. It was noted that this method is direct, accurate, objective, and fast. In one publication, this method was used to determine the antioxidant capacity of olive oil, i.e., fat-soluble samples, from different Mediterranean countries-Italy, Greece, France, Spain, Morocco, Tunisia [38]. This method allows for the assessment of quality and authenticity of olive oil. In one study, the antioxidant power of lipophilic compounds present in vegetables, such as carotenoids, chlorophyll, tocopherols and capsaicin, was determined [39]. When applied to pure compounds, the order of antioxidant power resulted as follows (in descending order): lycopene $>$ beta-carotene $>$ zeaxanthin $>$ alpha-carotene $>$ beta-cryptoxanthin $>$ lutein $>\alpha$-tocopherol $>$ capsaicin $>$ chlorophyll $\mathrm{a}>$ chlorophyll $\mathrm{b}>$ astaxanthin $>$ canthaxanthin. The results of the antioxidant activity measurements obtained for five vegetable and two fruit extracts were compared to those obtained by the ABTS. A good correlation between the two methods was found, except for spinach. The authors conclude that the amperometric method can be successfully used for direct, quick, and reliable monitoring of antioxidant power of lipophilic food extracts. Amperometric (electrochemical) method is also used to determine the antioxidant status in people. 


\section{Results of Antioxidant and Antiradical Activity of Coffee}

\subsection{Antioxidants and Antioxidant Activity}

The most antioxidant-rich beverages are [1]: coffee-200-550 mg/cup; tea-150-400 mg/cup; red wine - $150-400 \mathrm{mg} /$ glass. Intake of these drinks makes a significant contribution to the total amount of antioxidants consumed by people.

Green coffee beans contain large amounts of polyphenolic antioxidants, such as chlorogenic, caffeic, ferulic, and $n$-coumarinic acids. Coffee roasting significantly alters the composition of polyphenols due to Maillard reaction (chemical reaction between amino acids and sugars).

A cup of coffee which contains $10 \mathrm{~g}$ of roasted coffee beans may have 15 to $325 \mathrm{mg}$ of chlorogenic acids. On average in America, one cup contains approximately $200 \mathrm{mg}$ of chlorogenic acid. Antioxidant activity of ferulic and caffeic acids was studied both in vitro and in vivo. Ferulic acid presented in coffee has anti-inflammatory, anti-allergic, antibacterial, antiplatelet, and antiviral effect [34]. Pharmacological properties of ferulic acid are related to its high antioxidant activity, in particular, its ability to inhibit lipid peroxidation in biological membranes.

In one study, it has been shown that ferulic acid at a concentration of $10^{-3} \mathrm{~mol} / \mathrm{L}$ in a perfusion solution reduces arrhythmia [40].

People consume coffee or tea every day, sometimes several times a day. Coffee is the main drink in Europe, America and Asia. In recent years, consumption of coffee has increased even in England, a prominently tea-drinking country. Coffee consumption is rising rapidly in China and Japan where a centuries-old tradition of tea consumption is strongest.

Coffee and tea provide a very significant portion of the daily intake of antioxidants established for humans.

We have compared coffee, tea and cocoa in terms of the total antioxidant content (TAC). The results of the comparison are shown in the Table 2.

Table 2. Total antioxidant content of different beverages. Note: Natural roasted coffee produced by the Madeo Company. Best grades of green and black tea purchased in the stores. (Averaged values of TAC) [41].

\begin{tabular}{ccc}
\hline Beverages & One-time consumption rate, in grams & TAC, $\mathbf{~ g} / \mathbf{g}$ \\
\hline Coffee & $7-10$ & $150-300$ \\
Green Tea & 2 & $150-300$ \\
Black Tea & 2 & $110-200$ \\
Cocoa & 10 & $200-250$ \\
\hline
\end{tabular}

The data presented in Table 2 suggest that in terms of antioxidant capacity (as total antioxidant content), coffee and cocoa are comparable with tea, which can be attributed to higher consumption rates of these products. The antioxidant capacity of tea is largely related to the catechins and their polymers (thearubigins, etc.).

Cocoa is the most balanced product in terms of antioxidants since it contains water-soluble and lipid-soluble antioxidants. This point towards an interesting combination of coffee and cocoa and some manufactures are attempting to create such mixtures. 
Table 3 summarizes data obtained in Italy on the antioxidant activity of various non-alcoholic and alcoholic beverages [42]. It should be noted that figures provided for green and black tea as well as for beer are understated. According to our data, the antioxidant activity of cognac is higher than that of whisky.

Table 3. Comparison between the antioxidant activity (AA) of coffee and other drinks [42] (Notes: All samples were collected in Italy; FRAP —-ferric reducing antioxidant power; TRAP — total radical-trapping antioxidant parameter; TEAC - trolox equivalent antioxidant capacity).

\begin{tabular}{ccccc}
\hline \multirow{2}{*}{ No. } & Beverages & \multicolumn{3}{c}{ AA determined by different methods } \\
\cline { 3 - 5 } & FRAP $\left(\mathbf{m o l ~ F e} \mathbf{~ F}^{2+} \mathbf{L}\right)$ & TRAP $(\mathbf{m o l}$ Trolox/L) & TEAC (mol Trolox/L) \\
\hline 1. & Coffee (Espresso) & 129.4 & 66 & 36.5 \\
2. & Coffee (Instant) & 108.6 & 52.4 & 32.5 \\
3. & Coffee (Extract) & 96.4 & 59.6 & 30.3 \\
4. & Coffee (Espresso, Decaffeinated) & 93.0 & 45.8 & 27.0 \\
5. & Red wine (Chianti) & 31.5 & 14.8 & 11.4 \\
6. & Green Tea & 18.0 & 7.6 & 6.0 \\
7. & Black Tea & 10.1 & 4.9 & 3.6 \\
8. & Rose wine (Villa Tofre) & 8.3 & 2.2 & 2.4 \\
9. & White wine (Pinot) & 3.7 & 2.1 & 1.7 \\
10. & Whisky & 3.4 & 2.3 & 1.7 \\
11. & Cognac & 2.2 & 1.5 & 1.3 \\
12. & Beer & 2.8 & - & 1.0 \\
\hline
\end{tabular}

Data of caffeine, theobromine, and total polyphenols content in standard cups of coffee and tea, as well as their antioxidant activity, are provided in Table 4 [20].

Table 4. Caffeine, theobromine and total polyphenols content in one cup of coffee and tea [20]. (Notes: Caffeine and theobromine were determined using high performance liquid chromatography (HPLC); the total content of polyphenols was determined by Folin-Ciocalteu assay).

\begin{tabular}{cccc}
\hline No. & Description & Coffee & Tea \\
\hline 1. & Caffeine, $\mathrm{mg} /$ cup & $181 \pm 10$ & $130 \pm 7$ \\
2. & Theobromine, mg/cup & $28.9 \pm 1.1$ & $5.9 \pm 0.4$ \\
3. & The total content of polyphenols, gallic acid equivalents (GAE) $\mathrm{mg} / \mathrm{cup}$ & $161 \pm 9$ & $87 \pm 9$ \\
4. & AA, Crocin test, $\mathrm{mM}$ TX equivalent/cup & $7.4 \pm 0.3$ & $1.4 \pm 0.1$ \\
5. & TRAP, $\mathrm{mm} \mathrm{ROO}^{\circ}$ equivalent/cup & $10.1 \pm 0.6$ & $1.3 \pm 0.1$ \\
\hline
\end{tabular}

Table 5 shows the effect of the roasting degree of Arabica and Robusta coffee beans on their antioxidant activity [11]. The antioxidant activity of the beverage is represented by the increase of the lag time (lag time of LDL in the presence of coffee beverage with respect to the lag time of control $\mathrm{LDL}$ ). Results are expressed as mean (of three to five individual experiments) $\pm \mathrm{SD}$. 
Table 5. Antioxidant activity of green and roasted Arabica and Robusta coffee beans [11].

\begin{tabular}{cccc}
\hline \multirow{2}{*}{ No. } & Type of Coffee & \multicolumn{2}{c}{ Increase of lag time, min } \\
\cline { 3 - 4 } & & Arabica & Robusta \\
\hline 1. & Green & $366 \pm 74$ & $643 \pm 68$ \\
& Roasting degree: & & \\
2. & - light & $284 \pm 80$ & $294 \pm 41$ \\
3. & - medium & $206 \pm 30$ & $190 \pm 39$ \\
4. & -dark & $168 \pm 23$ & $134 \pm 34$ \\
\hline
\end{tabular}

As can be seen from Table 5, the antioxidant activity of Robusta green coffee is significantly higher than that of Arabica. However, this difference virtually disappears after light roasting; and after dark roasting Arabica coffee even exceeds Robusta coffee with regards to antioxidant activity.

In one article, antioxidant activity of coffee, tea, and cocoa - the most widely consumed beverages containing polyphenolic antioxidants-was compared [11]. The beverages were prepared as $0.7 \%-2.5 \%$ soluble coffee and 1.5\%-3.5\% cocoa; teas (green, black, or herbal) were prepared as one tea bag brewed in $220 \mathrm{~mL}$ of hot water. These drinks are often taken with milk. It has been shown that addition of milk did not alter the antioxidant activity.

The currently used roasting methods - convection and microwave roasting — were compared in [17].

Data on the antioxidant activity measured by spectrophotometric (UV-vis) and HPLC methods, as well as data on caffeine content in various types of coffee and in coffee roasted by different methods, is presented in Table 6 .

Table 6. Total polyphenols and caffeine content of raw and different roasted coffee beans [17].

\begin{tabular}{ccccc}
\hline \multirow{2}{*}{ No. } & Type of coffee and roasting method & \multicolumn{2}{c}{ Total amount of polyphenols, \% } & \multirow{2}{*}{ Caffeine, $\%$} \\
\cline { 3 - 4 } & GV & HPLC & 2.12 \\
\hline 1. & Green & 7.88 & 7.15 & \\
& Roasted: & & & 1.82 \\
2. & - convection & 2.26 & 2.07 & 1.90 \\
3. & - microwave & 2.31 & 2.19 & 1.89 \\
4. & - wet, convection & 2.58 & 2.36 & 2.07 \\
5. & - wet, microwave & 2.75 & 2.64 & 1.86 \\
6. & - dry, convection & 2.06 & 1.97 & 2.08 \\
7. & - dry, microwave & 2.11 & 2.03 & 1.95 \\
\hline 8. & - convectio microwave & 3.03 & 2.87 & \\
\hline
\end{tabular}

As can be seen from Table 6, microwave roasting preserves antioxidants better than convection roasting, and the wet method for separating the beans from the fruits is preferable to the dry method by this criterion. The latter is probably related to the greater oxidation of the antioxidants in the dry method.

In one study, efficiency of extraction of biologically active compounds from the coffee powder was researched [43]. Extraction was carried out with ethyl acetate, ethyl ether, and dichloromethane. Ethyl acetate in acidic medium was shown to be the most effective.

The yellow-brown extract of Robusta coffee was separated by gel-filtration chromatography according to the molecular weight. Individual fractions were collected at the column outlet for further study of 
their antioxidant activity (using a $\beta$-carotene-lipoic acid model system). Protective activity (PA) against lipid oxidation was determined in vitro. The results of these studies are summarized in Table 6.

As can be seen from Table 7, only high-molecular weight fractions showed antioxidant activity and only low-molecular weight fractions had protective activity. These interesting data require further careful research.

Table 7. Dry residue, molecular masses, antioxidant activity (AA\%), and protective activity (PA\%) of coffee fractions obtained by gel filtration chromatography (GFC) of acidic extract [43].

\begin{tabular}{cccccc}
\hline No. & Sample & $\mathbf{m g} / \mathbf{m L}^{\mathbf{a}}$ & Molecular weight & $\mathbf{A A}, \mathbf{\%}$ & $\mathbf{P A}, \boldsymbol{\%}$ \\
\hline 1. & acidic coffee extract & 1.31 & - & 96 & 100 \\
2. & 1st fraction & 0.50 & $<6000$ & 74 & 0 \\
3. & 2nd fraction & 0.16 & $<550$ & 37 & 0 \\
4. & 3rd fraction & 0.13 & $350-550$ & 0 & 0 \\
5. & 4th fraction & 0.23 & $200-350$ & 0 & 53 \\
6. & 5th fraction & 0.29 & $<200$ & 0 & 85 \\
\hline
\end{tabular}

Note: ${ }^{a} \mathrm{mg}$ of dry residue per $\mathrm{mL}$ of brewed coffee.

In that same study, the $\mathrm{pH}$ of the coffee solutions (green and varying degrees of roasting) was also determined. Robusta coffee varieties from Zaire and Ecuador and Arabica coffee varieties from Brazil, Colombia, and Costa Rica were studied. According to the results of this research, all varieties of green coffee have a higher $\mathrm{pH}$ than roasted coffees. Values ranges from 5.77 to 5.95. For roasted Arabica coffee varieties the $\mathrm{pH}$ values were lower than for Robusta varieties: 5.01-5.39 and 5.43-5.68, respectively. As for the antioxidant activity, it is higher in green coffee than in roasted; at the same time, for all coffee varieties its value increased by $4 \%-6 \%$ with the increase of the roasting time (from 10 to $30 \mathrm{~min}$ ).

\subsection{Antiradical Activity}

It should be emphasized that there is a difference between "antiradical" and "antioxidant" activity. The antiradical activity characterizes the ability of components to react with free radicals (in a single free radicals reaction), but antioxidant activity represents the ability to inhibit the process of oxidation (which usually involves a set of different reactions) [44].

In [45] have been shown that solutions of green and roasted coffee have significant antiradical activity against hydroxyl free radicals. This effect was studied both in vitro (using deoxyribose assay) and in vivo in biological cellular systems (IMR32 cells).

All solutions tested in vitro using the deoxyribose degradation assay have shown similar inhibitory activity. In the cell cultures, solutions of Robusta roasted coffee have shown greater activity than other solutions. Using preparative gel-filtration chromatography, the components of the roasted Robusta coffee were separated into three fractions by their molecular weight. The most active fraction was found to be the one containing the 5-O-caffeoylquinic acid. Activity of the solution increased when concentration of this acid increased (from 0.02 to $0.10 \mathrm{mM}$ ).

When roasted, the content of chlorogenic acids decreases but at the same time, the content of high molecular compounds, and melanoidins (also possessing antiradical activity in coffee) increases. 
In order to confirm that the 5-O-caffeoylquinic acid has the greatest efficiency, solutions of coffee biologically active compounds were prepared and tested for their antiradical activity (see Table 8).

Table 8. Antiradical activity in vitro and in vivo of standard solutions of coffee biologically active components [45].

\begin{tabular}{cccc}
\hline \multirow{2}{*}{ No. } & \multirow{2}{*}{ Compounds } & \multicolumn{2}{c}{$\%$} \\
\cline { 3 - 4 } & & in vitro & in vivo \\
\hline 1. & $5-O$-caffeoylquinic acid & $34.6 \pm 7.5$ & $264.3 \pm 56.2$ \\
2. & Caffeine & $27.2 \pm 6.4$ & $25.2 \pm 5.2$ \\
3. & Nicotinic acid & $19.2 \pm 6.7$ & $23.2 \pm 4.8$ \\
4. & Trigonelline hydrochloride & $19.3 \pm 12.6$ & $15.7 \pm 4.1$ \\
\hline
\end{tabular}

Table 8 shows that 5 - $O$-caffeoylquinic acid has the highest activity both in vitro and in vivo, particularly, in vivo. These results could explain the significant neuroprotective effects found for coffee consumption in recent epidemiological studies. These studies have established a direct link between coffee consumption and reduced risk of neurodegenerative diseases, including Parkinson's disease [46-48].

Coffee and coffee beverages can inhibit in vitro the mutagenicity of oxidants, such as tert-butyl hydroperoxide, and therefore can inhibit lipid peroxidation and formation of malondialdehyde, a marker of oxidative stress [49].

\subsection{Total Antioxidant Content by Amperometric Method}

The amperometric measurements of total antioxidant content has been provided by amperometric detector or analyzer TsvetYauza-01-AA (Khimavtomatika, Moscow, Russia).

The device operates on specially selected modes whereby only antioxidants are determined, and other compounds do not interfere with their determination.

Samples to be used for the determination of the total content of antioxidants in coffee were prepared as follows: the roasted coffee beans were ground into a powder, then boiling water was added to the powder, and the mixture was brewed for 5-10 min. A sample was taken using a syringe and introduced to a six-way valve on the device. When the valve was turned from the "dosing" position to the "analysis" position, the mobile phase (eluent) captured the sample and transferred it to the cell of the amperometric detector.

The total antioxidant content (TAC) in roasted coffee beans (provided by coffee manufacturer Freshly Roasted Company; coffee varieties of the MADEO trademark) from major coffee producers in 21 countries, is shown in Table 9. Quercetin was used as a reference substance.

As can be seen from Table 9, the content of antioxidants in different varieties of coffee differs 1.5 times at most and depends on the country of origin, degree of roasting, coffee processing method, altitude at which the coffee was grown, the soil, the average temperature, and number of sunny days per year.

Drinking two or three cups of coffee made with roasted beans ensures the daily consumption rate for antioxidants. Unfortunately, only $20 \%$ of consumers use natural roasted coffee in Russia, whereas, for example, in the U.S., this figure is $80 \%$. In recent decades, instant coffee grades have become widely used. True lovers of coffee, "coffee nerds", undoubtedly prefer natural roasted coffee because this type of coffee has a unique taste and aroma. 
Table 9. The total content of antioxidants (TAC) in MADEO coffee beans provided by company "Freshly Roasted Coffee" (quercetin used as standard) [41,50].

\begin{tabular}{cccc}
\hline No. & Name & TAC, $\mathbf{~ g / g}$ & $\begin{array}{c}\text { TAC, mg/7 g } \\
\text { (per cup) }\end{array}$ \\
\hline 1 & Maragogype, Guatemala & 32.1 & 224.7 \\
2 & Brazil & 30.8 & 215.6 \\
3 & Colombia & 30.5 & 213.5 \\
4 & Yirgacheffe, Ethiopia & 28.6 & 200.2 \\
5 & Puerto Rico & 28.6 & 200.2 \\
6 & Decaffeinated coffee & 27.3 & 191.1 \\
7 & Kenya & 27.0 & 189.0 \\
8 & Cuba & 26.7 & 186.9 \\
9 & Jamaica & 26.1 & 182.7 \\
10 & Sumatra & 24.5 & 171.5 \\
11 & Mexico & 24.1 & 168.7 \\
12 & Nicaragua & 24.1 & 168.7 \\
13 & Guatemala & 24.0 & 168.0 \\
14 & Honduras & 23.9 & 167.3 \\
15 & Maragogype, Nicaragua & 23.2 & 162.4 \\
16 & Maragogype, Mexico & 23.1 & 161.7 \\
17 & Tanzania & 23.1 & 161.7 \\
18 & Yemen & 22.9 & 160.3 \\
19 & Costa Rica & 22.5 & 157.5 \\
20 & Skybury, Australia & 22.3 & 156.1 \\
21 & Java & 21.1 & 147.7 \\
\hline
\end{tabular}

Due to some concentration during the extraction procedure, some varieties of instant coffee may contain greater amounts of antioxidants than roasted coffee.

The TAC values for green and roasted coffee beans from several different countries in Africa, South America, and Asia are provided in Table 10. As can be seen from Table 9, Arabica roasted coffee beans showed higher TAC than green coffee beans, which is quite an unexpected result. Previously, similar results were observed in some studies which used other methods.

Table 10. Impact of coffee roasting process on the total antioxidant content (quercetin used as standard) $[41,50]$.

\begin{tabular}{ccccc}
\hline \multirow{2}{*}{ No. } & \multirow{2}{*}{ Type of Coffee } & \multirow{2}{*}{ Producing Country } & \multicolumn{2}{c}{ TAC, mg/g } \\
\cline { 4 - 5 } & & Green & Roasted \\
\hline 1. & Arabica & Tanzania & 34 & 43 \\
2. & Arabica & Ethiopia (Yirgacheffe) & 32 & 40 \\
3. & Arabica & Brazil & 30 & 41 \\
4. & Robusta & Bali & 65 & 63 \\
5. & Robusta & Uganda & 67 & 55 \\
\hline
\end{tabular}




\section{Conclusions}

In the publications reviewed, the values for both antioxidant activity (AA) and total antioxidant content (TAC) of roasted coffee are at the same level as corresponding values for tea, cocoa, and red wine.

Roasting has a contradictory effect on TAC and AA. The HPLC results show that the content of chlorogenic acids, the main antioxidants in green coffee beans, significantly decreases after roasting. On the other hand, in the process of roasting such polymeric compounds as melanoidins (which are potent antioxidants) and other compounds are formed.

\section{Conflicts of Interest}

The authors declare no conflict of interest.

\section{References}

1. Menshchikova, E.B.; Lankin, V.Z.; Zenkov, N.K. Oxidative Stress: Pro-Oxidants and Antioxidants; Slovo: Moscow, Russia, 2006.

2. Menshchikova, E.B.; Zenkov, N.K.; Lankin, V.Z.; Bondar, I.A.; Trufakin, V.A. Oxidative Stress: Pathological Conditions and Diseases; ARTA: Novosibirsk, Russia, 2008.

3. Yashin, Y.I.; Ryzhnev, V.Y.; Yashin, A.Y.; Chernousova, N.I. Natural Antioxidants: Their Content in Foods and Impact of Human Health and Aging; TransLit: Moscow, Russia, 2009.

4. Harman, D. Aging: Minimizing free radical damage. J. Anti-Aging Med. 1999, 2, 15-36.

5. Khavinson, V.K.; Barinov, V.A.; Autyuryan, A.V.; Malinin, V.V. Free-Radical Oxidation and Aging; Russian Academy of Science: St. Petersburg, Russia, 2003.

6. Sies, H. Strategies of antioxidant defense. Eur. J. Biochem. 1993, 215, 213-215.

7. Matkovics, A. New strategies of antioxidant terapia. Orv. Hetil. 2006, 147, 747-752.

8. Shanin, Y.N.; Shanin, V.Y.; Zinoviev, E.V. Antioxidant Therapy in Clinical Practice; Albee Publishing House: Moscow, Russia, 2009; p. 128.

9. Belitz, H.D.; Grosh, W.; Schieberle, P. Coffee, Tea, Cocoa. Food Chemistry, New York; Springer-Verlag: Heidelberg, Berlin, Germany, 2009.

10. Rawel, H.M.; Kulling, S.E. Nutritional contribution of coffee, cacao and tea phenolics to human health. J. Verbraucherschutz Lebensmittelsicherheit 2007, 2, 399-406.

11. Richelle, M.; Tavazzi, I.; Offord, E. Comparison of antioxidant activity of consumed polyphenolic beverages (coffee, cocoa and tea) prepared per cup serving. J. Agric. Food Chem. 2001, 49, 3438-3442.

12. Nicoli, M.C.; Anese, M.; Manzocco, L.; Lerici, C.R. Antioxidant properties of coffee brews in relation to the roasting degree. Lebensm. Wiss. Technol. 1997, 30, 292-297.

13. Steinhart, H.; Luger, A.; Piost, J. Antioxidative Effect of Coffee Melanoidins. In Proceedings of the 19th International Scientific Collogue on Coffee, Trieste, Italy, 14-18 March, 2001.

14. Farah, A.; Donangelo, C.M. Phenolic compounds in coffee. Braz. J. Plant Physiol. 2006, 18, 23-36.

15. Fuster, M.D.; Mitchell, A.E.; Ochi, H.; Shibamoto, T. Antioxidant activities of the heterocyclic compounds formed in brewed coffee. J. Agric. Food Chem. 2000, 48, 5600-5603. 
16. Del Castillo, M.D.; Ames, J.M.; Gordon, M.H. Effect of roasting on the antioxidant activity of coffee brews. J. Agric. Food Chem. 2002, 50, 3698-3703.

17. Nebesny, E.; Budryn, G. Antioxidative activity of green and roasted coffee beans as influenced by convection and microwave roasting methods and content of certain compounds. Eur. Food Res. Technol. 2003, 217, 157-163.

18. Mullen, W.; Nemzer, B.; Stalmach, A.; Ali, S.; Combet, E. Polyphenolic and hydroxycinnamate contents of whole coffee fruits from China, India, and Mexico. J. Agric. Food Chem. 2013, 61, 5298-5309.

19. Mullen, W.; Nemzer, B.; Ou, B.; Stalmach, A.; Hunter, J.; Clifford, M.; Combet, E. The antioxidant and chlorogenic acid profiles of whole coffee fruits are influenced by extraction procedures. J. Agric. Food Chem. 2011, 59, 3754-3762.

20. Natella, F.; Nardini, M.; Giannetti, I.; Dattilo, C.; Scaccini, C. Coffee drinking influences plasma antioxidant capacity in humans. J. Agric. Food Chem. 2002, 50, 6211-6216.

21. Anese, M.; Nicoli, M.C. Antioxidant properties of ready-to-drink coffee brews. J. Agric. Food Chem. 2003, 51, 942-946.

22. Charurin, P.; Ames, J.M.; del Castillo, M.D. Antioxidant activity of coffee model systems. J. Agric. Food Chem. 2002, 50, 3751-3756.

23. Antolovich, M.; Prenzler, P.D.; Potsalides, E.; McDonald, S.; Robards, K. Methods for testing antioxidant activity. Analyst 2002, 127, 183-198.

24. Perez, D.; Leighton, F.; Aspee, A.; Aliaga, C.; Lissi, E. A comparison of methods employed to evaluate antioxidant capabilities. Biol. Res. 2000, 33, 1-10.

25. Llesuy, S.; Evelson, P.; Campos, A.M.; Lissi, E. Methodologies for evaluation of total antioxidant activities in complex mixtures. A critical review. Biol. Res. 2001, 37, 1-22.

26. Khasavov, V.V.; Ryzhova, G.L.; Mal’tseva, E.V. Antioxidants research methods. Chem. Plant Raw Mater. 2004, 3, 63-95.

27. Roginsky, V.; Lissi, E.A. Review of methods to determinate chain-breaking antioxidant activity in food. Food Chem. 2005, 92, 235-254.

28. Brainina, K.Z.; Ivanova, A.V.; Sharafutdinova, E.N. Academic news magazine. Potentiometric measurement of antioxidant activity of foodstuff. Food Technol. 2004, 4, 73-75.

29. Abdullin, I.F.; Turova, E.N.; Budnikov, G.K. Coulometric determination of the antioxidant capacity of tea extracts using electrogenerated bromine. J. Anal. Chem. Plant Raw Mater. 2001, 56, 627-629.

30. Cao, G.; Alessio, H.M.; Cutler, R.G. Oxygen-radical absorbance capacity assay for antioxidants. Free Radic. Biol. Med. 1993, 14, 303-311.

31. Gao, G.; Verdom, C.P.; Wu, A.; Wang, H.; Proir, R.L. Automated assay of oxygen radical absorbance capacity with the CORAS FARA II. Clin. Chem. 1995, 41, 1738-1744.

32. Ou, B.; Hampsch-Woodill, M.; Prior, R. Development and validated of an improved oxygen radical absorbance capacity assay using fluorescein as the fluorescent probe. J. Agric. Food Chem. 2001, 49, 4619-4626.

33. Hung, D.; Ou, B.; Hampsch-Woodill, M.; Flanagan, J.; Deemer, E. Development and validation of oxygen radical absorbance capasity assay for lipophylic antioxidants using randomly methylated-cyclodextrin as the solubility enhancer. J. Agric. Food Chem. 2002, 50, 1815-1821. 
34. Prior, R.L.; Hoang, H.; Gu, L.; Wu, X.; Bacchiocca, M.; Howard, L.; Hampsch-Woodill, M.; Huang, D.; Jacob, R. Assays for hydrophilic and lipophilic antioxidant capacity (oxygen radical absorbance capacioty $\left(\mathrm{ORAC}_{\mathrm{FL}}\right)$ ) of plasma and other biological and food samples. J. Agric. Food Chem. 2003, 51, 3273-3279.

35. Wu, X.; Beecher, G.; Holden, J.; Haytowitz, D.; Gerhard, S.; Prior, R. Lipophilic and hydrophilic antioxidant capacities of common foods in the united states. J. Agric. Food Chem. 2004, 52, 4026-4037.

36. Prior, R.; Wu, X.; Schaich, K. Standardized methods for the determination of antioxidant capacity and phenolics in foods and dietary supplements. J. Agric. Food Chem. 2005, 53, 4290-4302.

37. Mannino, S.; Brenna, O.; Buratti, S.; Cosio, M.S. A new method for the evaluation of the "antioxidant power" of wines. Electroanalysis 1998, 10, 908-912.

38. Mannino, S.; Buratti, S.; Cosio, M.S.; Pellegrini, N. Evaluation of the "antioxidant power" of olive oils based on a FiA system with amperometric detection. Analyst 1999, 124, 1115-1118.

39. Buratti, S.; Pellegrini, N.; Brenna, O.V.; Mannino, S. Rapid electrochemical method for the evaluation of the antioxidant power of some lipophilic food extracts. J. Agric. Food Chem. 2001, 49, 5136-5141.

40. Dyakov, A.A.; Perfilova, V.N.; Tyurenkov, I.N. Antiarrhythmic effect of ferulic acid. Bull. Arrhythmol. 2005, 39, 45-52 (in Russian).

41. Yashin, Y.I.; Chernousova, N.I.; Fedina, P.A.; Levin, D.A.; Mironov, S.A. Determination of antioxidants in coffee by an amperometric method. Beer Beverages 2009, 2, 45-47.

42. Pellegrini, N.; Serafini, M.; Colombi, B.; Del Rio, D.; Salvatore, S.; Bianchi, M.; Brighenti, F. Total antioxidant capacity of plant foods, beverages and oils consumed in italy assessed by three different in vitro assays. J. Nutr. 2003, 133, 2812-2819.

43. Daglia, M.; Papetti, A.; Gregotti, C.; Berte, F.; Gazzani, G. In vitro antioxidant and ex vivo protective activities of green and roasted coffee. J. Agric. Food Chem. 2000, 48, 1449-1454.

44. Tirzitis, G.; Bartosz, G. Determination of antiradical and antioxidant activity: Basic principles and new insights. Acta Biochim. Pol. 2010, 57, 139-142.

45. Daglia, M.; Racchi, M.; Papetti, A.; Lanni, C.; Govoni, S.; Gazzani, G. In vitro and ex vivo antihydroxyl radical activity of green and roasted coffee. J. Agric. Food Chem. 2004, 52, 1700-1704.

46. Chen, F.; Xu, K.; Petzer, J.P.; Staal, R.; Xu, Y.; Beilstein, M.; Sonsalla, P.K.; Castagnoli, K.; Castagnoli, N.; Schwarzschild, M. Neuroprotection by caffeine and A (2A) adenosine receptor inactivation in a model of Parkinson's disease. J. Neurosci. 2001, 21, 1-6.

47. Ascherio, A.; Zhang, S.M.; Herman, M.A.; Kawachi, I.; Colditz, G.; Speizer, F.; Willett, W. Prospective study of caffeine consumption and risk of Parkinson's disease in men and women. Ann. Neurol. 2001, 50, 56-63.

48. Ross, G.W.; Abbott, R.D.; Petrovitch, H.; Morens, D.; Grandinetti, A.; Tung, K.; Tanner, C.; Masaki, K.; Blanchette, P.; Curb, J.D.; et al. Association of coffee and caffeine intake with the risk of Parkinson's disease. J. Am. Med. Assoc. 2000, 283, 2674-2678.

49. Stadler, R.H.; Turesky, R.J.; Muller, O.; Markovic, J.; Leong-Morgenthaler, P. The inhibitory effects of coffee on radical-mediated oxidation and mutagenicity. Mutat. Res. 1994, 308, 177-190. 
50. Yashin, Y.I.; Nemzer, B.V.; Ryzhnev, V.Y.; Yashin, A.Y.; Chernousova, N.I.; Fedina, P.A. Creation of databank for content of antioxidants in food products by amperometric method. Molecules 2010, 15, 7450-7466.

(C) 2013 by the authors; licensee MDPI, Basel, Switzerland. This article is an open access article distributed under the terms and conditions of the Creative Commons Attribution license (http://creativecommons.org/licenses/by/3.0/). 